\title{
Un programa de rastreo de cáncer prostático se asoció a una disminución de la mortalidad por dicha causa
}

A prostate cáncer screening program was associated with a decrease in prostate cáncer mortality

Hugosson J y col. The Lancet 2010, Vol 11; 725-732

\section{Objetivo}

Evaluar si realizar rastreo de cáncer de próstata $(\mathrm{CP})$ con antígeno prostático específico (en inglés PSA) cada dos años reduce su mortalidad especifica.

\section{Diseño, lugar y pacientes}

Ensayo clínico aleatorizado con un seguimiento promedio de 14 años. Fueron incluidos 20.000 pacientes nacidos en la ciudad de Göteborg, Suecia, entre 1930 y 1944, con una edad máxima al ingreso de 69 años. Fueron exluidos quienes habían emigrado o tenían diagnostico de $\mathrm{CP}$ al momento de la aleatorización (1994).

\section{Intervención y medición de los resultados principales}

Los pacientes fueron aleatorizados en dos grupos de 10.000. A los asignado al grupo rastreo, se les envió invitaciones escritas para realizar la prueba del PSA cada dos años, hasta que alcanzaran los 69 años. Aquellos pacientes con valores de PSA que superaran el umbral establecido fueron invitados a realizar una evaluación consistente en un tacto rectal y una biopsia prostática, repitiéndonse esta evaluación ante cada nuevo valor de PSA alterado. El resultado principal fue la mortalidad acumulada por CP y el secundario, su incidencia acumulada.
Resultados principales

La adherencia a la estrategia fue del $76 \%$. A los 14 años, la incidencia acumulada de $\mathrm{CP}$ en el grupo asignado a rastreo fue $12,7 \%$ y la del grupo control, $8,2 \%$. El grupo asignado a rastreo tuvo una mortalidad por CP $44 \%$ menor que el control (ver tabla 1) estimándose que sería necesario someter a rastreo a 293 personas (IC95\% 177 a 799) para prevenir una muerte por CP.

Tabla 1:

\begin{tabular}{l|c|c|c|c}
\multicolumn{1}{c|}{ Resultados } & $\begin{array}{c}\text { Grupo control } \\
\mathbf{n}=9952\end{array}$ & $\begin{array}{c}\text { Grupo rastreo } \\
\mathbf{n}=9952\end{array}$ & $\begin{array}{c}\mathbf{R R A} \\
(\mathbf{I C 9 5 \% )}\end{array}$ & $\begin{array}{c}\mathbf{R R} \\
(\mathbf{I C 9 5} \%)\end{array}$ \\
\hline $\begin{array}{l}\text { Mortalidad por } \\
\text { cáncer prostático } \\
\text { a los 14 años }\end{array}$ & $0,9 \%$ & $0,5 \%$ & $0,4 \%$ & 0.56 \\
$(0,17$ a 0,64$)$ & $(0,39$ a 0,82) \\
\hline
\end{tabular}

RRA: reducción del riesgo absoluto. RR: riesgo relativo.

\section{Conclusiones}

Un programa de rastreo de CP (bianual) es aceptable en hombres mayores de 50 años, en términos de una reducción de la mortalidad por $\mathrm{CP}$ a los 14 años cercana a la mitad.

\section{Comentario}

Con una menor edad de los participantes al inicio del programa, un seguimiento más prolongado, un intervalo inter-rastreo menor que el de otros estudios, un umbral de PSA más bajo para la realización de biopsia, y un índice mayor de biopsias ( $93 \%$ vs. 30 a $40 \%$ en PLCO) este estudio mostró una reducción de la mortalidad por CP $(\mathrm{RR} 0,56)$ superior que otros previos como el ERSPC (RR 0,84) y el PLCO² (no había mostrado diferencias).

Una de sus debilidades metodológicas es haber realizado la invitación a los participantes luego de la aleatorización, lo que implica que la decisión de someterse a las reglas del ensayo clínico pudo haber estado condicionada por saber el grupo al que cada uno iba pertenecer.

Recordamos que junto con el subgrupo de pacientes del estudio ERSPC', cuyas edades estaban comprendidas entre los 55 y los 69 años, el estudio que hoy comentamos es el único que reportó beneficios en términos de reducción de la mortalidad por CP. Sin embargo, ninguno de los ensayos clínicos publicados hasta hoy han encontraron beneficios en término de reducción de la mortalidad global.

\section{Conclusiones de la comentadora}

Como amplían el artículo editorial ${ }^{3}$ y el metanálisis ${ }^{4}$ comentado en este en este mismo número de EVIDENCIA, es importante valorar los resultados de este ensayo en el contexto del cuerpo de conocimiento ya existente respecto de la efectividad de la estrategia del rastreo de CP.

Yamila Mahumud [ Servicio de Medicina Familiar y Comunitaria del Hospital Italiano de Buenos Aires. yamila.mahumud@hospitalitaliano.org.ar ]

Mahumud $Y$. Un programa de rastreo de cáncer prostático se asoció a una disminución de la mortalidad por dicha causa. Evid Act Pract Ambul 13(4) 126 Oct-Dic. 2010. Comentado de: Hugosson j y col. Mortality results from the Göteborg randomised population-based prostate-cancer screening trial. The Lancet 2010, Vol 11; 725-732. PMID: 20598634.

\section{Referencias}

1. Schroder F y col. Screening and prostate-cancer mortality in a randomized European study. N Engl J Med 2009;360:1320-8.

2. Andriole G y col. Mortality results from a randomized prostate-cancer screening trial. N Engl J Med 2009;360:1310-9.

4. Ciapponi A. Nueva Evidencia sobre el rastreo de cáncer prostático: ¿Nuevas recomendaciones? Evid. Act. Pract. Ambul. 13(4). XX-XX. Oct-Dic. 2010.

4. Ciapponi A. Nueva Evidencia sobre el rastreo de cáncer prostático: ¿Nuevas recomendaciones? Evid. Act. Pract. Ambul. 13(4). XX-XX. Oct-Dic. 2010.
5. Drimer C. Rastreo de cáncer de próstata: continúa la intertidumbre. Evid Act Pract Ambul. 13(4).XX. Oct-Dic 2010. Comentado de: Djulbegovic M y col. Screening for prostate 5. Drimer C. Rastreo de cáncer de próstata: continúa la intertidumbre. Evid Act Pract Ambul. 13(4).XX. Oct-Dic 2010. Comen
cancer: systematic review and metaanalysis of randomised controlled trials. BMJ 2010; 341:c4543 doi: 10.1136/bmj.c4543. 